\title{
Phenomena in Polarized Disperse Systems Caused by Exchange-Correlation Interaction between Particles
}

\author{
Vladimir F. Kharlamov \\ Oryol State University, Oryol, Russia \\ Email: vladkharl@rambler.ru
}

How to cite this paper: Kharlamov, V.F. (2020) Phenomena in Polarized Disperse Systems Caused by Exchange-Correlation Interaction between Particles. Journal of Applied Mathematics and Physics, 8, 1304-1317.

https://doi.org/10.4236/jamp.2020.87100

Received: June 8, 2020

Accepted: July 14, 2020

Published: July 17, 2020

Copyright $\odot 2020$ by author(s) and Scientific Research Publishing Inc. This work is licensed under the Creative Commons Attribution International License (CC BY 4.0).

http://creativecommons.org/licenses/by/4.0/

(c) (i) Open Access

\begin{abstract}
We have studied the self-consistent states of nano- and micro-particle polarized powders and structures consisting of parallel particle chains and have determined conditions under which the static dielectric permittivity of a disperse system is negative. It has been shown that in such system an electric current runs without ohmic losses. We present the arguments for the physics of spontaneous emergence of the electric field in disperse systems made up of electrically neutral particles. It has been determined the influence the phase boundaries of a disperse system has on the origin of spontaneous polarization state. The structures consisting of parallel chains of dielectric particles can exhibit spontaneous polarization. In this case the properties of the spherical structure are similar to those of the ball lightning. It has been established correspondence of the obtained theoretical results with the experimental data available in the literature.
\end{abstract}

\section{Keywords}

Nanostructures, Negative Static Dielectric Permittivity, Ohmic Losses, Spontaneous Polarization, Aerosols

\section{Introduction}

Spontaneous emergence of the electric field in ferroelectrics is a known effect. The atoms that form a ferroelectric domain reside in self-consistent states due to dipole-dipole interaction between them [1]. The nano and micro granules in electrically polarized disperse systems can reside in the similar states. Polarization of the granules brings about exchange-correlation interaction between them. Due to the presence of local fields in the bulk of a polarized non-uniform 
semiconductor, its charge carries can also reside in self-consistent states and form a macroscopic size system. The electrical physical properties of electrically polarized disperse systems have not been thoroughly studied.

For an electron-type semiconductor polarized by a sine-wave electric field with a frequency $\omega$, there is observed a phase shift between the temporal dependence of the electric field strength and related dependence of the non-equilibrium part of the electron distribution function. As a result, the real part of relative dielectric permittivity $\varepsilon^{\prime}$ in a semiconductor may become negative [2] [3] [4] [5]. In non-uniform granular semiconductors, due to the inequality $\varepsilon^{\prime}<0$, the dielectric loss is negative [4] [5]:

$$
\operatorname{tg} \delta=\frac{\varepsilon^{\prime \prime}}{\varepsilon^{\prime}}=\frac{\sigma}{\omega \varepsilon_{0} \varepsilon^{\prime}}<0
$$

where $\varepsilon^{\prime \prime}$ is the imaginary part of dielectric permittivity; $\sigma$ is the electrical conductivity; $\varepsilon_{0}$ is the electric constant.

The state of matter polarized by a sine-wave electric field depends on its dielectric permittivity $\varepsilon(\omega, \boldsymbol{g})$, where $\boldsymbol{g}$ is a wave vector. According to the Kramers-Kronig dispersion relation for the static dielectric permittivity, for any electrically polarized substance, one of the following relations holds: $\varepsilon \geq 1$ or $\mathcal{E} \leq$ 0 , where $\varepsilon=\varepsilon(0, \bar{g}) ; \omega=0 ; \bar{g}$ is the average value of the wave vector $g$, $\bar{g} \gg g_{0} ; g_{0} \sim 1 / L ; L$ is the macroscopic size of the system [6]. The inequality $\varepsilon<$ 0 arises from the presence of the local fields, i.e. from the difference between the effective field acting on the charged particles of the media and the mean macroscopic field relative to which the dielectric permittivity is determined [7]. The very existence of matter with a negative static dielectric permittivity $\varepsilon$ is not obvious. This issue has been a subject of discussion (see, e.g. [7]).

Theoretical estimates predict negative values of $\varepsilon(0, g)$ for large values of $g$ in the case of non-ideal plasmas. Examples of the systems with $\boldsymbol{\varepsilon}(0, \boldsymbol{g})<0$ at $\boldsymbol{g} \neq 0$ are a metallic hydrogen and a Wigner crystal. Negative values of $\varepsilon(0, g)$ are possible not only for model systems but can also occur in real world systems such as melted sodium chloride [6] [7].

There have been obtained semiconductor structures whose electrical properties have been interpreted using the condition $\varepsilon<0$, where $\varepsilon=\varepsilon(0, \bar{g})$ is the static dielectric permittivity [8] [9]. For example, this condition has been used to explain the zero ohmic loss for a direct current in $\mathrm{Al}_{2} \mathrm{O}_{3}$ and $\mathrm{NiO}$ powders containing donor centers on the nanoparticle surfaces in the form of interstitial hydrogen atoms [8]. The zero ohmic loss has also been observed for a sine-wave current in finely dispersed compounds $\mathrm{KMnO}_{4}$ [8] [10] and $\mathrm{KNaC}_{4} \mathrm{H}_{4} \mathrm{O}_{6} \cdot 4 \mathrm{H}_{2} \mathrm{O}$ [9] placed in molecular hydrogen and vacuum, respectively. In the case of the finely dispersed dielectrics $\mathrm{KMnO}_{4}, \mathrm{~Pb}\left(\mathrm{NO}_{3}\right)_{2}$ and $\mathrm{CsNO}_{3}$ with donor centers on the particle surfaces, there is observed spontaneous emergence of a macroscopic electric field [11] [12] [13]. This effect can result from mutual repulsion of opposite charges in the powder bulk due to the inequality $\varepsilon<0$. A spontaneously polarized $\mathrm{KMnO}_{4}$ powder serves as a direct current source that absorbs heat 
from the ambient environment [13].

In solids with a negative static dielectric permittivity $\mathcal{E}$, free electrons experience mutual attraction which, according to estimates, may bring about superconductivity at temperatures significantly exceeding $300 \mathrm{~K}$ [6] [7]. However, the structure details and properties of solids for the relation $\varepsilon<0$ to hold true have not been determined yet.

The aim of the present work is to carry out a theoretical study of conditions under which the static dielectric permittivity of the systems consisting of semiconductor and dielectric nano- and microparticles becomes negative. Besides, we have solved the problem of a theoretical description of the properties of such systems observed in the experiments [8]-[13].

\section{Semiconductors with Negative Static Dielectric Permittivity}

Polarization of a solid by an electric field changes its internal (potential) energy [14]. This change depends on the structure of the solid. Interaction between polarized structural units of a non-uniform solid has effect on its dielectric permittivity. Let us consider this effect. First we will define the conditions under which the static dielectric permittivity of a semiconductor becomes negative.

Consider an electric circuit that includes a current source, a switch, and a capacitor with a semiconductor placed between its plates (Figure 1). Upon closing the switch, the source begins to move the charges. The changing charge on the plates changes the electric field strength inside the capacitor.

According to Maxwell's equations, the alternating current density in a semiconductor consists of two terms:

$$
\boldsymbol{j}_{s}=\boldsymbol{j}+\frac{\partial \boldsymbol{D}}{\partial t},
$$

where $j$ and $\partial D / \partial t$ are the conduction and displacement current densities; $D=$ $\varepsilon \varepsilon_{0} E ; E$ is the electric field strength, and $t$ is time. Let at an arbitrary moment of time the following inequality hold

$$
j(t) \ll \frac{\partial D(t)}{\partial t} .
$$

Multiplying both sides of (1) by $E(t)$, we get

$$
j(t) E(t) \ll \frac{\partial F(t)}{\partial t},
$$

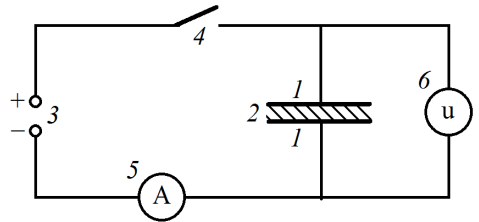

Figure 1. Electric circuit diagram. 1 capacitor plates; 2 finely dispersed semiconductor; 3 current source; 4 switch; 5 ammeter; 6 voltmeter. 
where

$$
F=\frac{D^{2}}{2 \varepsilon \varepsilon_{0}}=\frac{\varepsilon \varepsilon_{0} E^{2}}{2} .
$$

The quantity $F$ is the change of free energy of a unit volume of a dielectric polarized by an electric field in an isothermal process [14]. The left-hand side of (2) determines the ohmic loss, while the right-hand side describes the change of free energy of a solid. Therefore, in order for the polarization to determine the electrical properties of the semiconductor, the inequality (1) must be satisfied. It is a necessary but not a sufficient condition for the inequality $\varepsilon<0$ to be held.

According to expression (3), if $\varepsilon<0$, we have $F<0$. We will show that the conditions $F<0$ and $\varepsilon<0$ can be satisfied for finely dispersed (granular) semiconductors due to interaction between polarized particles by means of local electric fields. In this case the semiconductor particles are in self-consistent states.

Consider a powder with electronic conductivity of its particles. The process under which the system's free energy decreases $(F<0)$ occurs spontaneously [14]. We therefore assume that the particle local electric fields also emerge spontaneously. Transition of the powder into a polarized state can occur because of random self-consistent fluctuations of the dipole moments of the particles due to chaotic thermal motion of free electrons. Let us calculate the free energy $F$ and the corresponding value of $\varepsilon$ for a layer of spontaneously polarized powder placed between parallel capacitor plates.

The interacting charges of polarized particle dipoles reduce the powder internal energy. The electrostatic energy of interaction of $N$ charges $+q$ with $N$ charges $-q$, that make up the dipoles of the polarized powder, per unit volume is

$$
U_{e}=\frac{N q\left(\varphi^{+}-\varphi^{-}\right)}{2 V},
$$

where $U_{e}<0 ; N$ is the number of particles in the volume $V ; \varphi^{+}$and $\varphi^{-}$are the mean potentials of the self-consistent fields acting on respectively positive $+q$ and negative $-q$ charges. These potentials are produced by all charges of the system. In direct vicinity of a charge $q$, charges of the opposite sign are predominant. At a distance $r$ from a particle, the charge density at the location of other powder particle is

$$
\rho(r)=e\left(n^{+}-n^{-}\right)
$$

where $e$ is the electron charge; $n^{+}=n_{0} \exp \left[-e \varphi /\left(k_{B} T\right)\right]$ is the concentration of ionized donor centers; $n^{-}=n_{0} \exp \left[e \varphi /\left(k_{B} T\right)\right]$ is the concentration of free electrons; $n_{0}=m N / V$ is the mean concentration of the same sign charge carriers; $m$ is the number of free electrons in the powder particle volume; $k_{B}$ is the Boltzmann constant; $T$ is the temperature; $\varphi$ is the field potential determined from Poisson's equation

$$
\Delta \varphi(r)=-\rho(r) / \varepsilon_{0}
$$


Assuming that the condition $e \varphi \ll k_{B} T$ is true (a "weak" field case), we make use of the expression $\rho(r)=-2 e^{2} n_{0} \varphi(r) /\left(k_{B} T\right)$. Additionally, we perform averaging of the potential spatial dispersion over the media non-uniformity. Due to spherical symmetry of the field near each charge $q$, and since $\varphi(r) \sim 1 / r$, Poisson's equation can be approximately solved to give $\varphi(r)=(C / r) \exp (-c r)$, where $C$ is a constant; $c$ is a reciprocal value of the shielding length

$$
c=\left(\frac{2 e^{2} m N}{\varepsilon_{0} k_{B} T V}\right)^{1 / 2}
$$

The potential of the field produced by all charges except a given one $q$, at a distance $r$ from it, is $\varphi_{q}=(C / r) \exp (-c r)-q /\left(4 \pi \varepsilon_{0} r\right)$, and at the location of the charge itself at $r=0$, by expanding $\exp (-c r)$ in a row, we get

$$
\varphi^{+}=\lim _{r \rightarrow 0}\left[C \exp (-c r)-\frac{q}{4 \pi \varepsilon_{0}}\right] \frac{1}{r}=\lim _{r \rightarrow 0}\left(C-\frac{q}{4 \pi \varepsilon_{0}}\right) \frac{1}{r}-C c=-\frac{q c}{4 \pi \varepsilon_{0}},
$$

where $C=q /\left(4 \pi \varepsilon_{0}\right)$. Using the equality $\varphi^{-}=-\varphi^{+}$, we have:

$$
U_{e}=\frac{N q\left(\varphi^{+}-\varphi^{-}\right)}{2 V}=-\frac{q^{2} N C}{4 \pi \varepsilon_{0} V}
$$

Using expression (6), the Gibbs-Helmholtz equation $U=F-T(\partial F / \partial T)_{V}$ [14], and the condition $F \rightarrow 0$ at $q \rightarrow 0$, we obtain

$$
F=-\frac{q^{2} N c}{6 \pi \varepsilon_{0} V}
$$

The field strength in the bulk of spontaneously polarized powder is

$$
E_{p}=\frac{\sigma_{p}}{\varepsilon_{0}}=\frac{q}{\varepsilon_{0} l^{2}},
$$

where $\sigma_{p}$ is the surface charge density of opposite polarization charges on the opposite surfaces of the powder layers; $l$ is the particle diameter; $I^{2}$ is the number of particles on a unit surface of the powder layer. From (8), we find $q=\varepsilon_{0} P E_{p}$ and using this, the relation $F=\varepsilon \varepsilon_{0} E_{p} / 2$, and Formula (7), we get

$$
\varepsilon=-\frac{c l^{4} N}{3 \pi V} \approx-\frac{c l}{3 \pi},
$$

where $c$ is determined by (5); $N / V \approx I^{3}$.

Now let us make some estimates. Assuming in expressions (5), (7) - (9): $T=$ $300 \mathrm{~K} ; l=10^{-5} \mathrm{~m} ; E_{p}=5 \times 10^{3} \mathrm{~V} / \mathrm{m}$ (these values correspond to the experimental data [11] [12] [13]); $m=30$, we obtain: $N / V=10^{15} \mathrm{~m}^{-3} ; q=5 \times 10^{-18} \mathrm{C} ; c=2 \times$ $10^{5} \mathrm{~m}^{-1} ; \varepsilon=-0.2 ; F=-3 \times 10^{-5} \mathrm{~J} / \mathrm{m}^{3}$.

Thus according to (9), the static dielectric permittivity of a spontaneously polarized finely dispersed semiconductor is negative.

Note that the values $F$ and $E_{p}$ (see (7) and (8)) are bounded since the value of the polarization charges $q$, which depends on the concentration of free electrons in the powder particles, is bounded.

Let the powder consist of dielectric particles that contain on their surfaces the 
donor centers which by losing electrons produce a positive surface charge. Free electrons of the particles reside in the field produced by the positive surface charge and form a near-surface gas. It follows from Gauss's law as applied to a closed surface inside the powder particle. In this case, depending on the particle diameter, their bulk conductivity is small or zero; and when the powder is polarized by an external field, condition (1) is held. If a doping impurity (donor) is uniformly distributed over the volume of powder particles, their electrical conduction breaks condition (1). Additionally, because of the presence of the local fields in the bulks of polarized particles, their bulk conductivity brings about a decrease of their polarization charges, thereby imposing restriction on the spontaneous increase of $F$ and $E_{p}$ (see (7) and (8)). For this reason, the condition $\varepsilon<$ 0 is experimentally satisfied for dielectric powders containing the donor centers only on the particle surfaces. For powders with a bulk doping of their particles, the inequality $\varepsilon<0$ does not hold [8].

Thus, expressions (1) and (4) determine necessary conditions for the inequality $\varepsilon<0$ to be held in the case of powders. These conditions are satisfied for finely dispersed dielectrics with donor centers on the particle surfaces.

\section{Finely Dispersed Semiconductor in Electric Field}

The quantity $F_{a}=-F=-\varepsilon \varepsilon_{0} E^{2} / 2$ determines the change of free energy dielectric, when creating the field in the dielectric, taking into account its work against an external source that creates the field [14]. For a matter for which the condition $\varepsilon<0$ is true, when being acted on by a field, there arise internal forces that do work against the external source. In this case, the inequality $F_{a}>0$ corresponds to the appearance of electromotive force (EMF). In accordance with Le Chatelier's principle, the internal forces continuously pull the system back to its stationary state with a minimum potential energy $U_{e}$. The source of energy for EMF is the system's internal energy [13] [14].

It follows from Maxwell's equations that a variable field does not penetrate into the system with a negative dielectric permittivity $(E(t)=0)[2]$. Let us prove that the condition $E=0$ for a stationary external field $\left(E_{0}(t)=\right.$ const) follows from the inequality $\varepsilon<0$. Assume that on closing the switch (see Figure 1), the source produces an electric field in a semiconductor with $\varepsilon<0$. The first law of thermodynamics for an isothermal process of semiconductor polarization at constant volume will use

$$
A^{\prime}=U_{e}+Q,
$$

where $A^{\prime}>0$ is the source work to transport the charges that produce the field; $U_{e}$ is the change of the semiconductor potential energy after the polarization is completed; $Q$ is the amount of heat transferred to the thermostat. Integrating inequality (2), we get $Q \ll F$. According to the Gibbs-Helmholtz equation $U=F-T(\partial F / \partial T)_{V}$ we find: $U_{e}=0$, if $F=0$. So because of the conditions $A^{\prime}>0$ and $U_{e}<0$, equality (10) can be satisfied in the single case when

$$
A^{\prime}=0 ; U_{e}=0 ; Q=0 .
$$


Therefore, conditions (1) and (2) correspond to the equalities: $F=\varepsilon \varepsilon_{0} E^{2} / 2=0$; $E=0$. Ohmic losses is zero, $j(t) E(t)=0$, where $E(t)=0$, which corresponds to the condition $Q=0$.

Assuming that the process of semiconductor polarization is adiabatic, we have

$$
A^{\prime}=U_{e}+\Delta U_{T},
$$

where $\Delta U_{T}$ is the change of the internal energy of chaotic thermal motion in the solid, $\Delta U_{T}=Q$. Again, as in the case of isothermal process, we obtain

$$
E=0 \text {. }
$$

Thus when a semiconductor with a negative static permittivity is electrically polarized by a constant field, the field strength in the bulk of semiconductor vanishes. In this case, the conditions $E=0$ and $\varepsilon<0$ are interrelated and one may consider the equality $E=0$ as a condition for the static dielectric permittivity of a semiconductor to be negative.

Let us define the effect of the condition $E=0$ on thermal and electric phenomena in a solid with an electron-type conductivity.

In general, the electric flux $I_{e}=-j$ in a solid is determined by the equation [14]

$$
\frac{1}{\sigma} \boldsymbol{j}=\boldsymbol{E}+\alpha_{T} \operatorname{grad} T+\operatorname{grad} \frac{\xi}{e},
$$

where $j$ is the electric current density; $E$ is the electric field strength in the bulk; $\alpha_{T}$ is the thermal electromotive force; $\xi=\mu / N_{\mathrm{A}} ; \mu$ is the chemical potential of free electrons; $N_{\mathrm{A}}$ is the Avogadro constant. According to (12), there acts in the solid the field $\boldsymbol{E}_{G}$ of internal (thermodynamic) forces

$$
\boldsymbol{E}_{G}=-\alpha_{T} \operatorname{grad} T-\operatorname{grad} \frac{\xi}{e} .
$$

By a solid body here we understand a finely dispersed electron-type semiconductor. We will account for its granular structure by assuming that its static dielectric permittivity $\mathcal{E}$ is negative. Using (12) and (13), and assuming that the semiconductor is approximately uniform on a macroscopic scale, we plug $E=0$ into (12), and condition (12) will take the form

$$
\boldsymbol{j}=-\sigma \boldsymbol{E}_{G} .
$$

Basing on expression (14), we have, depending on mutual orientation of the vectors $\boldsymbol{j}$ and $\boldsymbol{E}_{G}$

$$
\begin{gathered}
\left(\boldsymbol{j}, \boldsymbol{E}_{G}\right)<0 ; \boldsymbol{j}=-\sigma \boldsymbol{E}_{G} . \\
\left(\boldsymbol{j}, \boldsymbol{E}_{G}\right)>0 ; j \rightarrow 0 ; \quad \sigma \rightarrow 0 .
\end{gathered}
$$

The current density has two values. If the vectors $j$ and $\boldsymbol{E}_{G}$ have the same direction then the current is caused by gradient of temperature or chemical potential gradient of the charge carriers (Equation (15)). In this case the ohmic loss is zero: $(j, E)=0$, with $E=0$. Condition (16) corresponds to a zero the electrical conductivity when the vectors $j$ and $E_{G}$ have the same direction. Hence, the powder transits from a conductive state to a non-conductive state when the current changes its direction to opposite. 
Conditions (14)-(16), being the consequence of the powder's negative dielectric permittivity $\varepsilon$, correspond to the experimental data obtained for the films consisting of spherical $\mathrm{Al}_{2} \mathrm{O}_{3}$ and (or) $\mathrm{Ni}$ nanoparticles with oxide layers of $\mathrm{NiO}$ on the surfaces [8]. The mean particle diameter was $100 \mathrm{~nm}$. First the chemisorption of hydrogen ions $\mathrm{H}^{+}$on the particle surfaces from the gas discharge hydrogen plasma was carried out. Then a strong field produced a built-in residual polarization field in the films. Due to the drift of interstitial protons $\mathrm{H}^{+}$in the particles under the field action, their concentration on the film surface in contact with the cathode increased, while decreasing on the opposite surface adjoined to the anode. Because of the difference in thermionic work functions on the opposite surfaces of the film, there arises the potential difference of the built-in field $\Delta \varphi_{a c}=\left(A_{a}-A_{c}\right) / e$ between anode and cathode. In the process, the cathode acquires a negative charge and the anode a positive one. A non-uniform distribution of the donor impurity (interstitial $\mathrm{H}$ atoms) in the film corresponds to the formation of the chemical potential gradient of the free electrons. As an example, we give a VAC of the film consisting of $\mathrm{Al}_{2} \mathrm{O}_{3}$ nanoparticles (Figure 2) [8].

In the conductive state (line 1), according to conditions (15), the powder current is due to the electron diffusion and in this case the ohmic loss is zero. The non-conductive state (line 2), after the circuit current direction change, corresponds to condition (16).

\section{Particle Chains in Electric Field}

Next we will show that the state of a disperse system polarized by an external electric field and the sign of its dielectric permittivity $\varepsilon$ can depend on its interphase boundaries. For the sake of an example, we consider a periodic structure, consisting of dielectric particle chains, polarized by an external electric field. The structure is in contact with two dielectric plates placed perpendicular to the chains (Figure 3(a)).

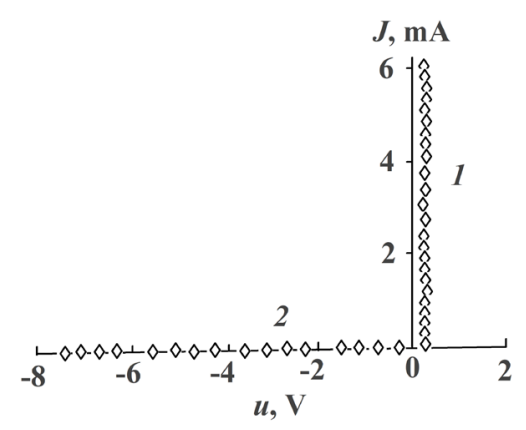

Figure 2. Dependence of the current through a film of alumina nanoparticles vs. magnitude and polarity of the voltage between the electrodes. 1 the electrical conductivity of the film is due to diffusion of electrons, ohmic losses are equal to zero (Formula (15)); 2 non-conductive state of the film (Formula (16)). The powder film was preliminarily treated in a hydrogen plasma for 15 minutes, then a built-in field was produced inside. The mean particle diameter $\bar{l}=100 \mathrm{~nm}$; layer thickness $d=10 \mu \mathrm{m} ; T=295 \mathrm{~K}[8]$. 


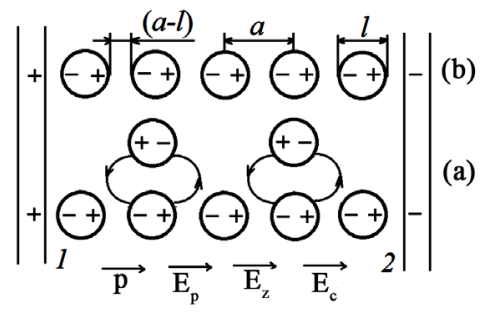

Figure 3. (a) Scheme the structure consisting of parallel chains of dielectric particles. The structure is in contact with dielectric plates 1 and 2; (b) "Dense" chain of spherical dielectric particles. $\boldsymbol{p}$ is the particle dipole moment; $\boldsymbol{E}_{c}$ is the electric field strength acting on a particle; $E_{p}$ is the field strength produced by the polarization charges located on the surfaces of the dielectric plates; $E_{z}$ is the local field produced by neighboring particles of the chain.

Consider first a single polarized "dense" chain of spherical dielectric particles separated by equal distances $a$, from each other (Figure 3(b)). Assume that the particle diameter $l$ is less than the separation: $0<(a-l)<l$. Let the chain be placed in a macroscopically uniform electric field with the strength vector $E_{0}$ directed along the chain. In this case, the local field of each polarized particle (sphere) outside its volume coincides with the field of the dipole located at its center and with the dipole moment parallel to the vector $\boldsymbol{E}_{0}$ [15]. The field strength of the dipole on its axis is $\boldsymbol{E}_{\boldsymbol{d}}=\boldsymbol{p} /\left(2 \pi \varepsilon_{0} a^{3}\right)$, where $\boldsymbol{p}$ is the particle dipole moment, $p=\alpha \varepsilon_{0} E_{s} ; \alpha$ is the polarizability; $E_{s}$ is the electric field strength acting on the particle. The total field produced by all polarized particles in the chain is

$$
\boldsymbol{E}_{s}=\frac{k \boldsymbol{p}}{2 \pi \varepsilon_{0} a^{3}}
$$

where $k$ is a coefficient, $k=2(1+1 / 8+1 / 27+1 / 64+\cdots)=2.4$. Due to interaction through their local fields, the polarized particles of the chain reside in self-consistent states.

The chains border on the dielectric plates on which surfaces the fields of the polarized chains induce local polarization charges $-q$ and $+q$ that have the opposite signs to those of the chain end charges $+q$ и $-q$ (Figure 3). These local polarization charges on the plates are not compensated and produce an additional field in the particle chain. Its field strength $E_{p}$ has the same direction as the vectors $E_{0}$ and $E_{s}$. So there arises a positive feedback between the surface polarization charges on the plates and the particle chain field. As a result, the local fields of the polarized chain particles increase, and we have in this case

$$
\boldsymbol{E}_{c}=\boldsymbol{E}_{s}+\boldsymbol{E}_{p}=\boldsymbol{E}_{s}+Y \boldsymbol{E}_{c}=\frac{\boldsymbol{E}_{s}}{1-Y},
$$

where $E_{c}$ is the field strength between the chain particles; $E_{p}=Y E_{\dot{c}} Y$ is a coefficient. Denote $1-Y=\varepsilon$. One can to the quantity $\varepsilon$ as a macroscopic characteristic of the chain of particles because of their interaction via the local fields they make up a unified system. The value $\varepsilon$ corresponds to the static dielectric permittivity of the particle chain. The condition $0<\varepsilon<1$ contradicts the energy conservation 
law. Hence if $Y>1$ (see below), we have $\varepsilon<0$. The inequality $\varepsilon<0$ is due to the exchange-correlation interaction of polarized particles by means of local fields and due to the positive feedback between the particle chain field and the polarization charges on the dielectric plates.

With increasing distance from the chain in the perpendicular direction, its field exponentially drops. At a distance of $2 a$, it decreases by $\exp (2 \pi)$ [1]. It means that mutual influence of "dense" chains of the structure (see Figure 3 ) is insignificant.

In the case of "sparse" chains, with a distance of $2 a$ between the particles (Figure 3(b)), the local fields at the distance $2 a$ are weak, so that $Y<1$ (see below) and the condition $\varepsilon<0$ not satisfied. Strengthening the field of the chain does not occur. On the other hand, the particles of "sparse" chains are in the field of the neighboring particles of the "dense" chains. In this case the lines of force of the local fields produced by the particles from neighboring chains have the same direction (see Figure 3(a)), which corresponds to the positive feedback between them. Owing to this, all chains of the periodic structure reside in self-consistent states and thereby form a unified system.

In experiments with the polarization of a finely dispersed semiconductor by a sine-wave field, its layer was placed between two dielectric plates, thus enabling the condition $\varepsilon<0$ to hold [9] [10].

\section{Spontaneous Polarization of Particle Chains}

The external field strength in a system with $\varepsilon<0$ vanishes (see (11)). So let us consider spontaneous polarization of the structure (Figure $3(\mathrm{a})$ ) resulting from spontaneous transition to a state with a minimum potential energy

$$
U_{e}=-\frac{N\left(\boldsymbol{p}, \boldsymbol{E}_{\boldsymbol{c}}\right)}{2 V}=-\frac{N p E_{c}}{2 V} .
$$

This transition occurs due to the positive feedback between the field strength $E_{c}$ and the polarization charges on the pates surface (see Equation (17)). Additionally, the spontaneous polarization is stimulated by the positive feedback between the local fields of polarized particles from neighboring "dense" and "sparse" chains.

Let us calculate the coefficient $Y$ in (17) for a "dense" chain of spherical dielectric particles (Figure 3(b)). For a dielectric ball polarized by a uniform electric field, the field potential at a surface point is [15]:

$$
\varphi=\frac{z P}{3 \varepsilon_{0}},
$$

where $z=(l \cos \theta) / 2 ; \theta$ is the angle between the polarization direction (chain axis) and the radius vector from the center of the ball to a surface point; $P$ is the ball polarization. According to (18), the potential difference between two points on the ball surface $\Delta \varphi$ depends only on the difference of their projections $z$ and $z_{1}$ on the chain axis, $\Delta \varphi=\left(z-z_{1}\right) P /\left(3 \varepsilon_{0}\right)$. If $\left(z-z_{1}\right)=1$, then $\Delta \varphi=I P /\left(3 \varepsilon_{0}\right)$. In a similar way, the potential difference between two points on the surfaces of two 
neighboring balls will depend only on the difference of their projections on the chain axis. For the potential difference between two surface points of two neighbor balls lying on the chain axis $(\cos \theta=1)$ separated by a distance $(a-l)$, we have

$$
\Delta \varphi_{c}=\frac{l P}{3 \varepsilon_{0}} .
$$

The surface charge density on a dielectric ball in a uniform electric field is distributed according to $\sigma_{s}=\sigma_{s m} \cos \theta$ [15]. Thus at $(a-l)<l$, the main contribution to the local field of polarized chain particles comes from small surface areas located near the chain axis. The particle surface charges produce local fields in between on the chain axis with the strength

$$
E_{z}=\frac{\Delta \varphi_{c}}{a-l}=\frac{l P}{3 \varepsilon_{0}(a-l)} .
$$

In the space between the particles the additional local field $E_{z}$ produced by the polarization charges has the same direction as the main field $\boldsymbol{E}_{\boldsymbol{s}}$. Hence, because of the boundary conditions the field increases. Substituting to (10) the expression [15]:

$$
P=\frac{3 \varepsilon_{0} E\left(\varepsilon_{w}-1\right)}{\varepsilon_{w}+2},
$$

where $E=E_{\dot{c}} \mathcal{E}_{W}$ is the ball dielectric permittivity, we get $E_{z}=Y E_{\dot{c}}$

$$
\begin{gathered}
Y=\frac{l\left(\varepsilon_{w}-1\right)}{(a-1)\left(\varepsilon_{w}+2\right)} ; \\
E_{c}=E_{s}+E_{z}=E_{s}+Y E_{c}=\frac{E_{s}}{1-Y}=\frac{E_{s}}{\varepsilon} .
\end{gathered}
$$

Using $E_{s}=k p /\left(2 \pi \varepsilon_{0} a^{3}\right)$, we have:

$$
E_{c}=\frac{k p}{2 \pi \varepsilon_{0} a^{3}(1-Y)},
$$

where

$$
p=\alpha \varepsilon_{0} E_{c} .
$$

The quantities $E_{c}$ and $p$ are connected through a positive feedback. Solving the system of Equations (21) and (22), we find the condition on the spontaneous polarization of the particle chain

$$
\alpha_{e f}=\frac{2 \pi a^{3}}{k},
$$

where $\alpha_{e f}=\alpha /|1-Y|$ is the particle effective polarizability. For $Y \approx 1 ; \alpha_{e f} \gg \alpha$, $\alpha \approx \beta$, so after a short-time action of an external field that polarizes the particles, the structure transits to a state of spontaneous polarization. In this case in (21), the condition $(1-Y)<0$ is held and it corresponds to the inequality $\varepsilon<0$. Setting, for example, $\varepsilon_{w}=81$ in (20) we find that the ratio a/l must be less than 
1.97. $Y \approx 1$ in $(21)$ corresponds to a sharp increase of the field strength. For $a=l$, according to (20) and (21), we have $E_{c}=0$.

The spontaneously polarized ball-shaped structure consisting of parallel particle chains produces an electric field which is uniform inside the ball volume and coincide with the dipole field outside (A polarized dielectric ball produces the field with similar lines of force [15]). Transition of the ball to the state of spontaneous polarization is accompanied by a change of its potential energy by the value

$$
U_{e}=-\frac{p E N}{2 V}=-\frac{\alpha_{e f} \varepsilon_{0} N E^{2}}{2 V}=-\frac{\pi \varepsilon_{0} a^{3} N E^{2}}{k V}
$$

where $p=\alpha_{e f \varepsilon_{0}} E$ is the particle dipole moment; $E$ is the field strength acting on the particle; $\alpha_{e f}$ is determined by Formula (23).

Expression (24) corresponds to a spontaneous increase in the field strength $E$. According to the Fowler-Nordheim equation, at the field strengths $E \geq 10^{9} \mathrm{~V} / \mathrm{m}$, there is observed the electron tunnel emission which results in the electric current between the chain particles. It imposes a restriction of $10^{9} \mathrm{~V} / \mathrm{m}$ on the value of $E$ due to increase in the electrical conductivity of the system. In this case, the ball electric field outside the bulk ignites an air corona discharge with a visible glow. This discharge is caused by the action of the ball EMF $G \approx E D$, where $D$ is the ball diameter. Because of an immense internal resistance of the source, the current and the discharge power are small. The closed electric circuit formed by the electron tunnel emission current between the ball particles and the corona discharge provides the balance of the system charges. This balance imposes further restrictions on the value of $E$.

In the ball bulk, the vectors $j_{V}$ and $E$ have opposite directions due to the condition $\varepsilon<0$. Because the inequality $\left(j_{n}, E\right)<0$ is satisfied, the heat is absorbed in the bulk. In this case, the work of the field in moving electrical charges outside the ball is done on the account of the system's internal energy. A similar effect is observed experimentally: a spontaneously polarized powder serves as a source of direct current [13].

A cloud of a polarized aerosol consisting of air-suspended disperse phase particles, which are drawn into the area of a strong field and which form chains, can have a ball shape with a diameter less than $1 \mathrm{~m}$. The fulfilment of condition (24) signifies a spontaneous aerosol transition to a state of a minimum potential energy as a result of its spatial self-organization which lies in the action of internal forces that drive the particles to the location where the condition $Y \approx 1$ is met. The minimum of the potential energy also stems from the spherical shape of the polarized cloud. The spontaneously polarized cloud is a source of an electric current in the form of corona discharge.

One may make an assumption that a ball lightning (BL) is a spontaneously polarized aerosol cloud of air-suspended water nanoparticles. Assuming that the strength of the uniform field inside a BL is $10^{9} \mathrm{~V} / \mathrm{m}$ and using Formula (24), we find that the BL energy density is $U_{e}=-10^{7} \mathrm{~J} / \mathrm{m}^{3}$. This value matches the maxi- 
mum energy density obtained from observing the BL explosions [16].

\section{Conclusions}

The static dielectric permittivity $\varepsilon$ of finely dispersed dielectrics with donor centers on the surface of particles has a negative value. The fulfillment of the condition $\varepsilon<0$ does not depend on the nature of the finely dispersed dielectric and the nature of donor centers. Due to the condition $\varepsilon<0$, an electron-type semiconductor powder transits to a state of spontaneous polarization with a diminished internal (potential) energy. Interaction of polarized powder particles brings about the emergence of self-consistent states of free electrons. In the process of the energy exchange occurring in the powder, the state of the whole electron system is subject to a change rather than the states of individual electrons.

The aerosols polarization can be accompanied by their spatial self-organization with a formation of particle chains in a disperse phase if the concentration of particles in the gas exceeds the critical value.

\section{Acknowledgements}

I thank V.A. Burdovizin for help in the work.

\section{Conflicts of Interest}

The author declares no conflicts of interest regarding the publication of this paper.

\section{References}

[1] Feynman, R.P., Leighton, R.B. and Sands, M. (1964) Feynman Lectures on Physics. Vol. 2, Addison-Wesley Publishing Company, London. https://doi.org/10.1063/1.3051743

[2] Bonch-Bruevich, V.L. and Kalashnikov, S.G. (1990) Semiconductors Physics. Nauka, Moscow, 450. (In Russian)

[3] Yan, H., Zhao, C., Wang, K., Deng, L., Ma, M. and Xu, G. (2013) Negative Dielectric Constant Manifested by Static Electricity. Applied Physics Letters, 102, Article ID: 062904. https://doi.org/10.1063/1.4792064

[4] Zhang, L., Chen, S., Yuan, S., Wang, D.R., Hu, P.-H. and Dang, Z.-M. (2014) Low Dielectric Loss and Weak Frequency Dependence of the Dielectric Constant of $\mathrm{CeO}_{2} /$ Polystyrene Nanocomposite Films. Applied Physics Letters, 105, 2905. https://doi.org/10.1063/1.4892456

[5] Axelrod, E., Puzenko, A., Haruvy, Y., Reisfeld, R. and Feldman, Y. (2006) Negative Dielectric Loss Phenomenon in Porous Sol-Gel Glasses. Journal of Non-Crystalline Solids, 352, 4166-4173. https://doi.org/10.1016/i.jnoncrysol.2006.07.008

[6] Bulaevskiy, L.N., Ginsburg, V.L., Zharkov, G.F., Kirzhniz, D.A., Kopaev, Yu.V., Maksimov, E.G. and Khomskiy, D.I. (1977) The Problem of High Temperature Superconductivity. Nauka, Moscow. (In Russian)

[7] Maksimov, E.G. and Dolgov, O.V. (2007) A Note on the Possible Mechanisms of High-Temperature Superconductivity. Physics-Uspekhi, 50, 933-937. https://doi.org/10.1070/PU2007v050n09ABEH006213 
[8] Kharlamov, V.F. (2019) Electrical Conductivity of Films with Negative Dielectric Permittivity. Journal of Surface Investigation: X-Ray, Synchrotron and Neutron Techniques, 13, 1306-1313. https://doi.org/10.1134/S1027451019060351

[9] Rogov, A.P. and Kharlamov, V.F. (2019) Nano- and Microstructures with Equal Zero Ohmic Losses in a Spontaneously Polarized State. Nanotechnologies in Russia, 14, 197-203. https://doi.org/10.1134/S1995078019030108

[10] Kharlamov, V.F. (2017) Enhancement and Inversion of an Alternating-Current Electric Field in a Finely Dispersed Dielectric. Physics Solid State, 59, 43-46. https://doi.org/10.1134/S1063783417010152

[11] Sokolov, A.A., Sergeev, V.O. and Kharlamov, V.F. (2017) Spontaneous Polarization of Hydrogen-Saturated Composite Materials. Russian Physics Journal, 59, 1460-1465. https://doi.org/10.1007/s11182-017-0931-Z

[12] Kharlamov, V.F., Sergeev, V.O. and Sokolov, A.A. (2016). Emergence of an Electric Field in Finely Disperse Dielectrics during Their Interaction with Hydrogen. Russian Journal of Physical Chemistry B, 10, 490-495.

https://doi.org/10.1134/S1990793116030246

[13] Kharlamov, V.F. (2018) Electromotive Force in a Layer of Finely Dispersed Spontaneously Polarized Semiconductor. Journal of Surface Investigation: X-Ray, Synchrotron and Neutron Techniques, 12, 1222-1225. https://doi.org/10.1134/S1027451018050592

[14] Bazarov, I.P. (1991) Thermodynamics. Vysshaya Shkola, Moscow. (In Russian)

[15] Purcell, E.M. (1971) Electricity and Magnetism, Berkeley Physics Course, Vol. 2. McGraw-Hill Book Company, New York.

[16] Smirnov, B. (1990) Physics of Ball Lightning. Physics-Uspekhi, 33, 261. https://doi.org/10.1070/PU1990v033n04ABEH002573 